\title{
Phenolic profiles of the endemic species Astragalus palibinii Polozhij
}

\author{
Olga Kotsupiy ${ }^{*}$, and Tatyana Shemetova \\ Central Siberian Botanical Garden, SB RAS, 630090 Zolotodolinskaya str., 101, Novosibirsk, Russia
}

\begin{abstract}
For the first time, the composition and content of phenolic compounds (PC) of plant leaves of an endemic rare species of the Xiphidium Bunge section of the genus Astragalus L. of Siberia Astragalus palibinii Polozhij was studied by HPLC. 11 compounds of phenolic nature were identified in water-ethanol extracts of A. palibinii leaves: gallic, p-hydroxybenzoic, chlorogenic, caffeic, ferulic, trans-ferulic, cinnamic acids, isoquercitrin, rutin, isoramnetin-3-O-rutinoside and Lepicatechin. The composition of $\mathrm{PC}$ in the leaves of plants of three cenopopulations $A$. palibinii from Khakassia is the same, with the exception of one hydroxycinnamic acid in trace amounts. The content (mg per $100 \mathrm{~g} \mathrm{DW}$ ) of flavonol glycosides in the leaves of A. palibinii plants varies in the range of $0.442-0.907$, the content of hydroxycinnamic and hydroxybenzoic acids in the range of 98.5-109.7 and 32.5-83.9, respectively. The quantitative profiles of all the studied groups of PC are characterized by significant variability in the content of individual components. The variability of the content of hydroxycinnamic acids, flavonoids and hydroxybenzoic acids was determined as very low, medium and increased, respectively.
\end{abstract}

\section{Introduction}

Astragalus palibinii Polozhij - polycarpic semi-shrub $4-15 \mathrm{~cm}$ tall with leaves $2.5-8 \mathrm{~cm}$ long, 5-7-paired, covered with white pressed hairs. The leaflets are narrowly linear, 4-10 $\mathrm{mm}$ long and 1-2 (3) $\mathrm{mm}$ wide, often with wrapped edges. The brushes are umbrellashaped. The corolla is white-pink or white with a flag 16-20 mm long. Beans are obliquely upward - standing, linear, slightly bent, 20-25 (35) mm long, 2-2.5 mm wide, keeled on the abdomen, grooved-on the back, leathery, white-pressed-hairy, 2-nesting, with a subulate spout up to $2 \mathrm{~mm}$ long [1-5].

A. palibinii is an endemic of the mountain-steppe belt of the northern part of the Eastern Sayan, areas of the Kansk, Abakan and Krasnoyarsk steppes. Xerophyte. It grows on open stony, gravelly and cartilaginous slopes, scree, in small-grain and meadow steppes. Blooms in late April, May. It does not bloom annually. In favorable years, it grows weakly. It grows in a limited area, in isolated locations, forms small populations [6].

*Corresponding author: olnevaster@gmail.com 
There is little information about the composition of phenolic compounds (PC) of plants of the Xiphidium section in literary sources. Kaempferol, quercetin, narcissin, populin, isoquercitrin, astragalin, isoramnetin-3-O- $\beta$-D-glucopyranoside were found in the plants of A. varius S. G. Gmelin growing in the European part of Russia [7]. The composition and content of PC in Asian species of the section has not been studied in detail before. There is information about the presence of flavonoids in the aboveground organs of A. suffruticosus DC [8].

The purpose of our work is to determine the phenolic profile of the endemic species $A$. palibinii, reflecting the composition and content of flavonol glycosides, hydroxycinnamic and hydroxybenzoic acids of plant leaves.

\section{Materials and Methods}

The study was carried out on the material collected during the phases of flowering - the beginning of fruiting on the territory of the Republic of Khakassia (Tab.). In each of the three cenopopulations (CP), an average sample was taken, collecting leaves from 7-10 individuals $(\mathrm{N}=7-10)$.

Table. Places, dates of collection and codes of the studied samples

\begin{tabular}{|c|c|}
\hline Code & Place and date of collection \\
\hline UAP16 & $\begin{array}{l}\text { Republic of Khakassia, Ust-Abakansky district, Podkuninsky mountains, north-western } \\
\text { slope, blackened steppe. } 53^{\circ} 54.186,091^{\circ} 24.388, \text { h }-287 . \text { T. A. Shemetova. } \\
09.07 .2016 \text {. }\end{array}$ \\
\hline UAM16 & $\begin{array}{l}\text { Republic of Khakassia, Ust-Abakansky district, shore of Krasnoyarsk reservoir. in the } \\
\text { vicinity of the v. of Mokhov, wormwood-feather grass steppe with a high } \\
\text { anthropogenic load. } 53^{\circ} 55^{\prime} 59^{\prime \prime}, 91^{\circ} 24^{\prime} 45^{\prime \prime}, \mathrm{h}-250 \text {. T. A. Shemetova. 13.07.2016 }\end{array}$ \\
\hline BeT10 & $\begin{array}{l}\text { Republic of Khakassia, Baysky district, in the vicinity of the v. Tabat, the southern } \\
\text { slope of the hill, forb-cereal steppe. } 52^{\circ} 58^{\prime}, 90^{\circ} 44^{\prime}, \text { h-309. D. N. Shaulo, A. A. Erst, T. } \\
\text { A. Myakshina. } 30.06 .2010\end{array}$ \\
\hline
\end{tabular}

For PC extraction, precisely weighted powdered air-dried plant material was exhaustively extracted with an ethanol:water mixture $(70: 30, \mathrm{v} / \mathrm{v})$ in a water bath at 60 $70^{\circ} \mathrm{C}$. For acid hydrolysis, $0.5 \mathrm{ml}$ of water-ethanol extraction was added to $0.5 \mathrm{ml}$ of $\mathrm{HCl}$ $(2 \mathrm{~N})$ and heated in a boiling water bath for 2 hours. The hydrolysate was cooled, diluted with distilled water, passed through a concentrating cartridge Diapak C16 («BioChemMac»), the aglycones were washed off with $96 \%$ ethanol. The eluates were filtered through a membrane filter with a pore diameter of 0.45 microns. The components were analyzed using an Agilent 1200 liquid chromatograph with a diode-matrix detector (Agilent Technologies, USA) and a ChemStation system for collecting and processing chromatographic data. The chromatographic separation was conducted at $25^{\circ} \mathrm{C}$ on a Zorbax SB-C18 Column $(4.6 \times 150 \mathrm{~mm}, 5 \mu \mathrm{m}$ i.d. $)$ in the gradient elution mode. The extracts and the hydrolysates were separated with gradient 1 and gradient 2 , respectively. In gradient 1 , methanol concentration in an aqueous solution of orthophosphoric acid $(0.1 \%)$ changed from 19 to $70 \%$ within 30 minutes, then to $100 \%$ by 32 minute, and in gradient 2 , the methanol concentration increased from 50 to $100 \%$ within 17 minutes. The flow rate was set to $1 \mathrm{~mL} \cdot \mathrm{min}^{-1}$. The sample injection volume was $10 \mu \mathrm{L}$, and the absorbance was measured at 210, 255, 270, 290, 325, 340, 360, and $370 \mathrm{~nm}$. Qualitative analysis of phenolic compounds was performed based on the peaks of reference standards of gallic, $\mathrm{p}$ hydroxybenzoic, chlorogenic, caffeic, ferulic, trans-ferulic, cinnamic acids, quercetin, kaempferol, isoramnetin, rutin, isoquercitrin, and isoramnetin-3-O-rutinoside (Sigma- 
Aldrich, St. Louis, MO, USA). Quantification of hydroxybenzoic and hydroxycinnamic acids was carried in equivalents of gallic and chlorogenic acids, respectively; of flavonol glycosides in rutin equivalents. The concentrations of the PC were expressed in mg per 100 $\mathrm{g}$ of absolutely dry weight (DW). Each sample was analyzed in three replicates.

All the data were processed in the Statistica 10.0 software (Statsoft Inc., Tulsa, OK, USA) and MS Excel, were reported as mean \pm standard error (SE) of three replicates, and were compared using ANOVA followed by Duncan's multiple range test. Differences between the means were considered statistically significant at the 5\% level $(\mathrm{p}<0.05)$. Evaluation of variability was carried out according to the absolute mean values of the studied sign and the value of the coefficient of variation $(\mathrm{Cv}, \%)$, taking into account the scale of variability levels proposed by S. A. Mamaev [9]: very low - less than 7; low - 715; average - 15-25; increased - 26-35; high - 36-50; very high - more than 50 .

\section{Results}

Rutin, isoquercitrin and isoramnetin-3-O-rutinoside were identified in the composition of the flavonoid glycosides of the leaves of $A$. palibinii, as well as ten unidentified substances with absorption maxima in two wavelength ranges 250 and 350-360 nm were also found. The hydrolysates contain quercetin, isorhamnetin and trace amounts of kaempferol. The composition of hydrolysates indicates that unidentified substances are flavonols glycosides [10]. In the three studied $A$. palibinii $\mathrm{CP}$, the composition of flavonols glycosides was identical, the content of individual components and their amounts varied (Fig. 1). The content of G13, G14 and Gl6 glycosides in water-ethanol extracts from plant leaves did not

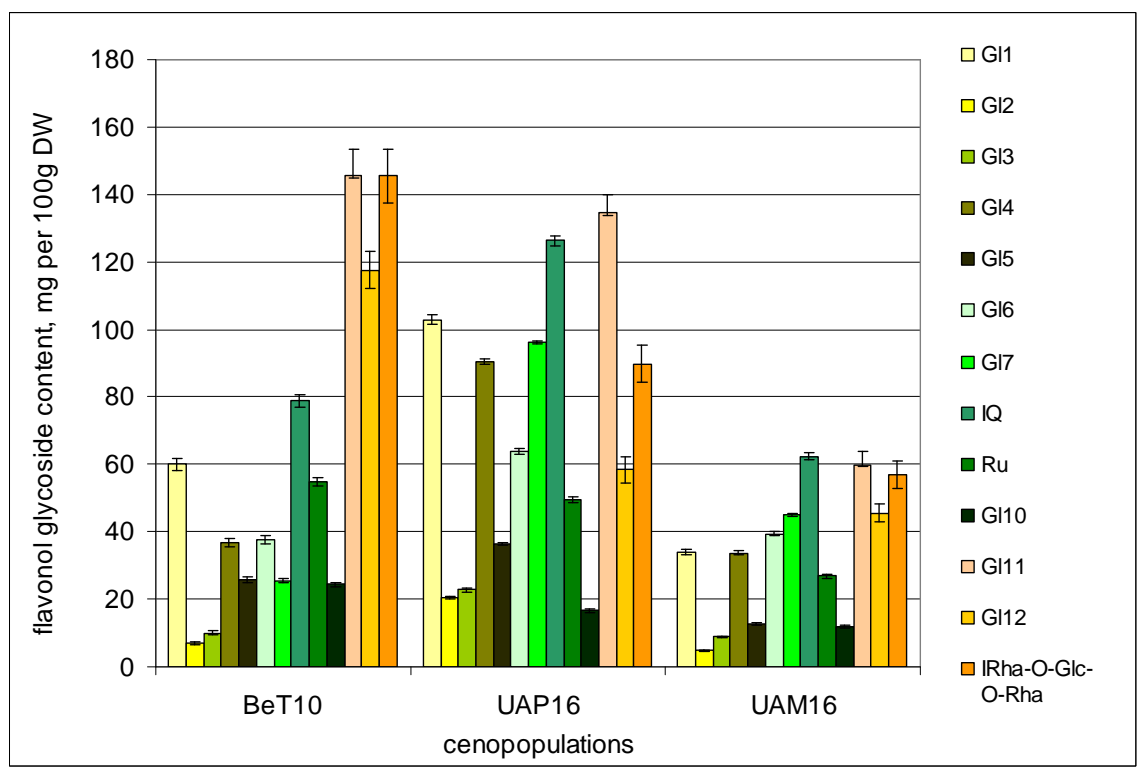

Fig. 1. Flavonoid profiles of A. palibinii leaves. Symbols: IQ - isoquercitrin, Ru - rutin, IRha-O-GlcO-Rha - isoramnetin-3-O-rutinoside, Gl1 - Gl12 - flavonol glycosides. BeT10, UAP16, UAM16 codes of cenopopulations (see tab. 1).

significantly differ in CP BeT10 and UAM16, the content of Gl11 glycoside in CP BeT10 and UAP16. The content of all other individual glycosides and the sum total of glycosides differed significantly in the plants of the studied CP. The sum of 13 flavonoid glycosides was (mg per $100 \mathrm{~g} \mathrm{DW}$ ) for CP BeT10, UAP16 and UAM16 769.5 $\pm 6.8,907.7 \pm 26.3$ and 
$442.0 \pm 3.0$, respectively. The ratio of the content of individual components, the so-called "flavonoid profile", was characterized by the greatest similarity in the UAP16 and UAM16 from the Ust-Abakansky district of Khakassia, differing twice in the total content of substances. The coefficient of variability of the content of flavonol glycosides in waterethanol extracts of plant leaves of three $\mathrm{CP}\left(\mathrm{C}_{\mathrm{v}}\right)$ was $24.0 \%$, which corresponds to the average level of variability.

The content of the remaining PC was determined by groups - hydroxybenzoic acids, hydroxycinnamic acids, catechins (Fig. 2). Gallic and p-hydroxybenzoic acid were identified in the composition of hydroxybenzoic acids. The content of the sum of three hydroxybenzoic acids significantly differed in all studied CP (BeT10 - 32.5 \pm 1.6 , UAP16 $66.9 \pm 1.8$, UAM16 - 83.9 $\pm 1.4 \mathrm{mg}$ per $100 \mathrm{~g} \mathrm{DW})$. A similarity was noted in the quantitative ratio of hydroxybenzoic acids in plant extracts of CP BeT10 and UAP16. Based on UV spectra and comparison with standard samples, chlorogenic, caffeic, ferulic, trans-ferulic and cinnamic acids were found among 14 hydroxycinnamic acids. The total sum of hydroxycinnamic acids in the extracts of plant leaves of the three CP did not differ significantly $(98.5 \pm 5.4,106.9 \pm 3.6$ and $109.7 \pm 4.0 \mathrm{mg}$ per $100 \mathrm{~g} \mathrm{DW})$. The profiles of hydroxycinnamic acids are more heterogeneous in the ratio of components than the profiles of hydroxybenzoic acids. Only one representative of catechins has been identified - Lepicatechin. Its content does not significantly differ in extracts of leaves of plants CP BeT10 and UAP16. The increased coefficient of variability $\left(\mathrm{C}_{\mathrm{v}}\right)$ characterizes the CP of $A$. palibinii according to the signs of the content of hydroxybenzoic acids and L-epicatechin 30.4 and $25.9 \%$, respectively; very low variability was found in the content of hydroxycinnamic acids $-3.9 \%$.

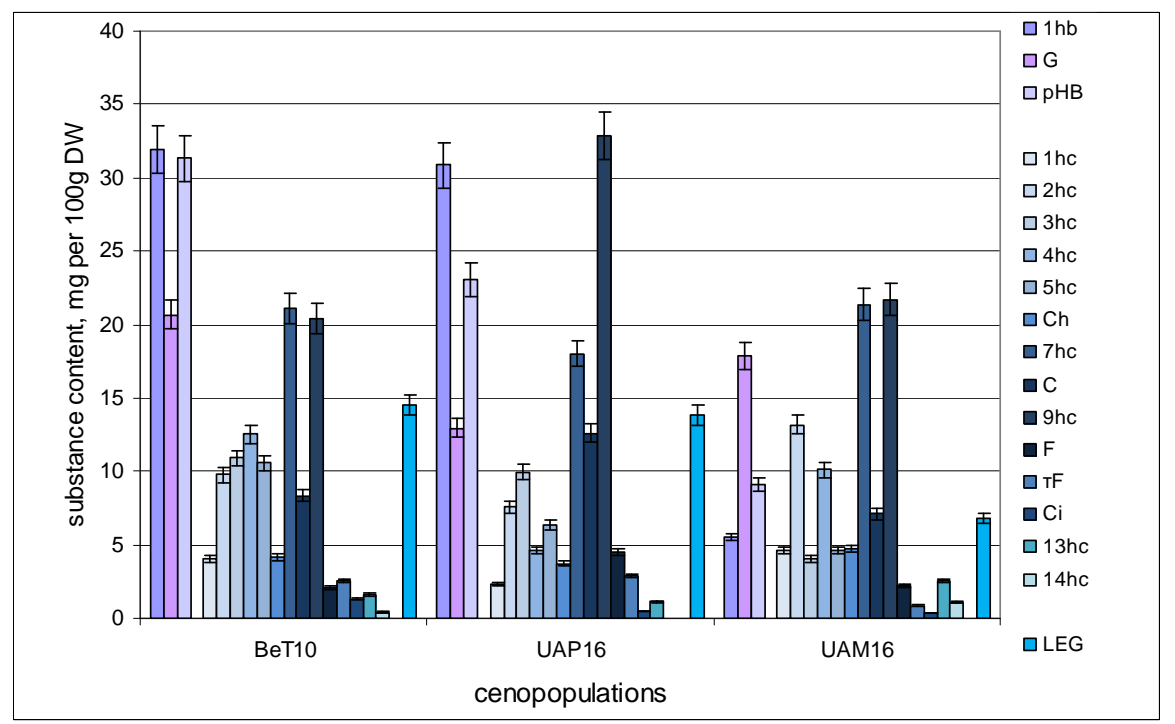

Fig. 2. Hydroxybenzoic, hydroxycoric acids and L-epicatechin of A. palibinii leaves. Symbols: G gallic acid, pHB - p-hydroxybenzoic acid, $\mathrm{Ch}$ - chlorogenic acid, $\mathrm{C}$ - caffeic acid, $\mathrm{F}$ - ferulic acid, $\mathrm{tF}$ - trans-ferulic acid, Ci - cinnamic acid, LEG - L-epicatechin, 1hb - hydroxybenzoic acid, 1hc-14hchydroxycinnamic acids.

\section{Discussion}

The composition of the PC of the leaves of the endemic A. palibinii is quite diverse and includes phenolic acids, flavonol glycosides and catechins. For the first time, gallic, p- 
hydroxybenzoic, chlorogenic, caffeic, ferulic, trans-ferulic and cinnamic acids, rutin, isoquercitrin and isoramnetin-3-O-rutinoside, as well as L-epicatechin were found in the leaves of this rare species. In the $3 \mathrm{CP}$ of Khakassia, the composition of 13 flavonol glycosides and 3 hydroxybenzoic acids remained unchanged, among 14 hydroxycinnamic acids, one minor component was found to be absent in CP UAP16. The coefficient of variability $\left(\mathrm{C}_{\mathrm{v}}\right)$ of the substance content is not the same for the FC groups. Thus, a very low level of variability was revealed in the total content of hydroxycinnamic acids, while the profiles in different CP have differences in the content of individual components. The sum total of flavonol glycosides varies at an average level of variability with differences in the content of individual glycosides. The sum total of hydroxybenzoic acids and the content of L-epicatechin have an increased value of the coefficient of variability.

\section{Conclusion}

The composition and content of PC in the leaves of plants of the endemic A. palibinii of the Xiphidium Bunge section were studied by HPLC. A qualitative and quantitative comparison of flavonoid profiles, profiles of hydroxycinnamic and hydroxybenzoic acids in waterethanol extracts of leaves of A. palibinii plants from three CP of Khakassia was carried out. Isoquercitrin, rutin and isoramnetin-3-O-rutinoside were identified in the composition of flavonol glycosides. Among the hydroxybenzoic compounds, gallic and p-hydroxybenzoic acid were identified, and among the hydroxycinnamic acids, chlorogenic, caffeic, ferulic, trans-ferulic, and cinnamic acid were identified. The composition of PC in the three CP does not change, with the exception of one hydroxycinnamic acid in trace amounts. The quantitative profiles of all the studied groups of PC are characterized by significant variability in the content of individual components, while the total content of hydroxycinnamic acids, flavonoids and hydroxybenzoic acids (98.5-109.7, 442.0-907.7, 32.5-83.9 mg per $100 \mathrm{~g} \mathrm{DW}$, respectively) have very low, medium and increased levels of variability $\left(\mathrm{C}_{\mathrm{v}}\right)-3.9,24.0,30.4 \%$, respectively.

The work was financially supported by the draft State assignment of the Central Siberian Botanical Garden of the Siberian Branch of the Russian Academy of Sciences No. AAAA-A21-121011290025-2 within the framework of a government contract and with material of CSBG representing USFs (Unique Scientific Facilities) "Collections of living plants indoors and outdoors" USU 440534.

\section{References}

1. A. V. Polozhiy, Flora of the Krasnoyarsk Territory, 6 (1960)

2. P. N. Krylov, Flora of Western Siberia, 7 (1933)

3. N. F. Goncharov, A. G. Borisova, Flora of the USSR, 12 (1946)

4. A. V. Polozhiy, Flora Island prieniseyskih steppes (Tomsk, 2002)

5. S. N. Vydrina, Flora of Siberia, 9 (1994)

6. E. M. Antipova, Red Book of the Krasnoyarsk Krai: Rare and Endangered Species of Wild Plants and Fungi, 2 (2012)

7. N. N. Guzhva, M. S. Lukyanchikov, A. L. Kazakov, Chem. of nat. comp., 6 (1987)

8. M. N. Bobrova, Questions of pharmacognosy, 1 (1961)

9. S. A. Mamaeev, Individual ecological and geographical variability of plants, 94 (1975)

10. M. N. Zaprometov, Fundamentals of the biochemistry of phenolic compounds: textbook (Moscow, Higher School, 1974) 